in the etiology of edema in this disease. Moreover, it has been reported that prolactin restores the aldosterone ${ }^{3)}$ and $\mathrm{ADH}^{4)}$ action in the escape state, and renal kallikrein-kinin system may be related to the escape phenomenon ${ }^{5}$. Therefore, higher plasma ADH levels and aldosterone concentration in the upright position may contribute to the development of edema via hyperprolactinemia and suppression of the renal kallikrein-kinin system.

On the basis of the present study, it could be assumed that the increased vascular permeability and the endocrinological poly factors were related in a cooperative fashion to the etiology of the idiopathic edema.

\section{REFERENCES}

1) Horrobin DF et al: Action of prolactin in human renal function. Lancet II : 352, 1971.

2) Mills IH et al: The renal kallikrein-kinin system and the regulation of salt and water excretion. Fed Proc 35: 181, 1976.

3) Burstyn PG et al: Saluretic action of aldosterone in the presence of increased salt intake and restoration of normal action by prolactin and oxtocin. J Endocrinol 55: 369, 1972.

4) Horrobin DF et al: Water losing action of antidiuretic hormone in the presence of excess cortisol : restoration of normal action by prolactin or by oxytocin. J Endocrinol $58: 135$, 1973.

5) Carretero OA and Scicli AG: Renal kallikrein : its localization and possible role in renal function. Fed Proc 35: 194, 1976.

\title{
II. Clinics of Edema (6) Treatment-Diuretics, Refractory Edema
}

\author{
Nobuhiro Sugino, MD
}

\author{
Department of Internal Medicine Tokyo Women's Medical College
}

Among diuretics, loop diuretics (LD) has been widelly used for patients with refractory edema. Short time test with furosemide (FM) has been applied to edematous patients, mainly renal and cardiac, and urine output and urinary solute excretion were measured.

1. Diuretic response to FM and GFR;

Following FM administration into eight young healthy men who have been in dry state overnight, negative free water clearance ( $T_{\mathrm{H} 2 \mathrm{O}}^{\mathrm{C}}$ ) decreased both under waterload and water deprivation. Same examinations were carried out to edematous patients with lower renal function. In three patients with chronic nephritis, whose Gcr was average $35.6 \mathrm{ml} / \mathrm{min}, \mathrm{T}_{\mathrm{H} 2 \mathrm{O}}^{\mathrm{C}}$ was unchanged under water-load but decreased slightly under water deprivation. In four nephritic patients with further lower renal function of mean Ccr $10 \mathrm{ml} / \mathrm{min}, \mathrm{T}_{\mathrm{H} 2 \mathrm{O}}^{\mathrm{C}}$ was not altered either under water-load or water deprivation. Therefore, it is concluded that, although urine output increases temporarily following LD administration, absence of response in free water clearance to the diuretics tends to indicate refractoryedema. 
(6) Treatment-Diurətics, Refractory Edema

2. Other measures to treat refractory edema; In addition to LD, other drugs are used with diuretics: antihypertensive to hypertensive diseases, peripheral vasodilatators to cardiac diseases, plasma volume expander to nephrotics and antialdosterone to cirrhosis. To patients with severe refractory edema and no absolute response to drug therapy, peritoneal and hemodialysis, parti- cularly with no dialysate flow (dialysate free ultrafiltration) are recommended. Ascites to jugular vein perfusion technic is also applied to refractory cirrhotic patients with severe ascites. However, these mechanical procedures produce only temporary improvement and overall outcome is dependent on recovery of original diseases. 